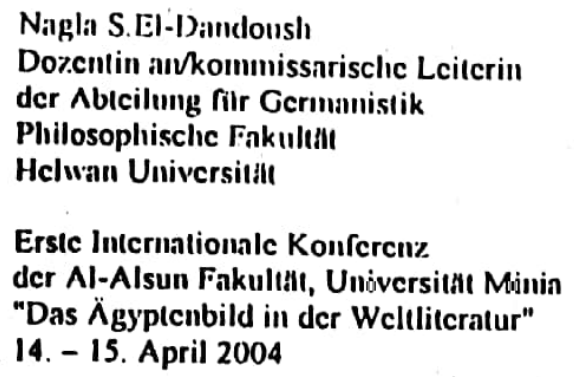

\title{
Zum Glauben und zur Mentalität der Alten Ägypter in Gerhard Armanskis Roman Die geflïgelte Sonne in Edfu. Roman aus dem prolemäischen ïgy'pten.
}

Der inn Jahlure 2000 erschienene Roman' seclne zovar in cler Tradition des historischen Romans über $\ddot{A}$ gypten, den bahabrechliend in Deutschlannd Gcorg Ebers mit scinem Roman, „Eine ägyptische Künigstochlıter cingeführt hat, crzählt dennoch dic Geschichte der Menschen am Nil im Rahmen der alı̈̈gyptischen Göltcrwelt aus cincr subjckliven, fikliven Perspcktive. Eipisoden aus dem modernen Ägypten werden in den Roman eingeblendet und weisen cinc Parallele zu der angeblich unverïnderten, statischıcn Mentalität dè Ägypter auf, dic sich über dic Jalurlauscnde hinweg nicht viel gewandelt haben soll - was zu überprüfen scin wird. Bemerkenswerl ist, wic licfgründig und bildlich der Autor Gerhard Armanski? dic ägyptische Welt von gesicrn darstcllt und Zusammenhlïnge zu Ägypten liculc herstellt.

Auffällig ist auch, daß der Roman nicht nur übcr alltägyptische Glaubens- und Mentalitätsgrundsailze aussagl, sondern sich anch zu curopäisch-antikcn Ġlaubcns- und Mcotalitätsanıs̈̈lzcı äußcrt. Beide werden šıch gegentibersicllt und der l.eser dazu angeregh, sich scincn Teil dabci z.u denken. Dic Darstellung von Kulturgeschichte und cinc gevvisse

\footnotetext{
' Ziticn wird im folgenden nach Arminski, Gerhard: Dic geflïgclıc Somnc von Lidfu. Reminn ams dem plolcmatisclicn Ägyplen. Würzburg 200). Zitate aus dem Roman werden mil der Sigle (Eiffi) und der Scilcuzalil nachgewiescn

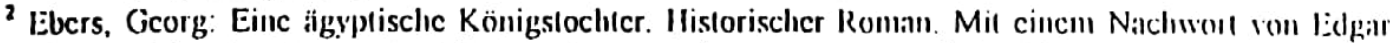

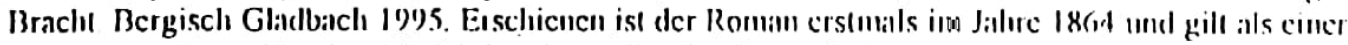

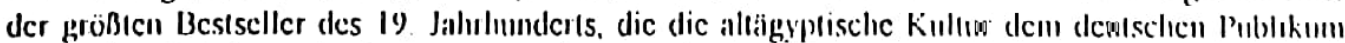

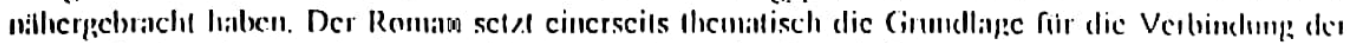

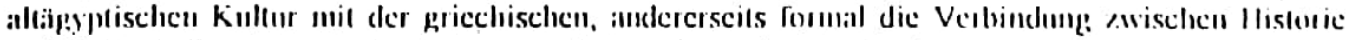

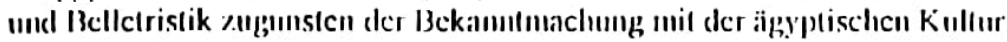


Mentalitätsforschung im litcrarischen Rahmen zcichnen ihn aus - wic noch zı zeigen scin wird. Es wäre nicht verkelırt zu sagen, daß̣ der Roman sozialkritisch angelegt ist. Demnoch wird ein schr licbevolles Bild von Ägypten gemalı, das ebenso wie in Ebers „Eine Ägyptische Königstochter" Interesse für das Land weckt und dazu bewegt, das Geheimnisvolle am Land aufzuspüren!

Da das Buch relativ neu ist, und keine Forschungsliteratur dazu vorhanden ist, bedient sich die Verfasserin im vorliegenden Beitrag einer durchgängig werkimmanenten Textanalyse. Viel Raum für Interpretation gab natürlich der ägyptische Hintergrund, sei es der historische oder der landesbezogene.

Im Mittelpunkt des Romans steht Hor-Heb, ein Diener dritten Grades ' des Golles Horus von Edfu, den jährlích seine Gemahlin, Hathor von Dendera, festlich besucht. Hor-Heb und parallel dazu der Tempelhüter Abderrachman, ein armer Diener der Polizeiwache, erleben die Welt der' großen Götter aus der Position der Untergebenen, aber überzeugten Diener des ,großen Gottes“ heraus, auch weinn jeder einen anderen Gott anbetet. Hor-Heb, was in der wörtlichen Übersetzung aus der pharaonischen Sprache „Horus ist glücklich“4 bedeutet, koreliert mit dem Naınen des Polizeiwächtes Abderrachman, welches in der iuchstäblichen Übersetzung aus der arabischen Sprache „Diener des Barmherzigen“ heißt.

Der Roman eröffnet wie folgt, um die Größe der Götterwelt versus der Beschränktheit des kleinen Lebens ihrer Diener zı zeigen:

Abderrachman erfuhr den Tempel des großen Gottes, der insoweit mit Allah verwandt sein mußte, als glücklafte Wende in seinem Leben, das so ganz nach niederer Polizcicharge in einem oberägyptischen [...] Dorf gerochen hatte. (E.dfu $5)$.

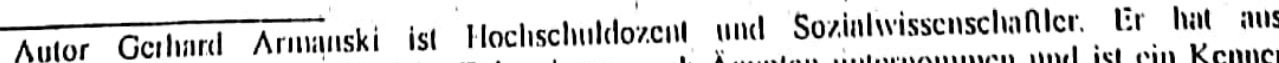

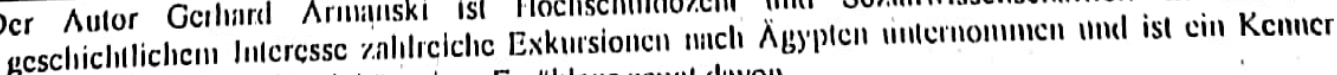

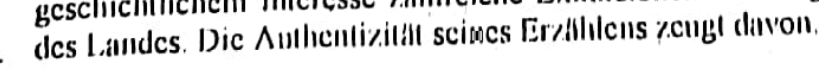

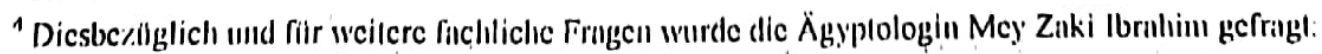


Hinter dieser Sprache verbirgt sich keine ketzerische Absicht, auch nicht in der Herstellung des Zusammenhangs zwischen dem Anbeten eines großen Gottes damals und Allah heute. Vielmehr drückt sich hier ein Vergleich zwischen ähnlichen Lebensumständen sozialer Art aus, die sich über Jahrtausende hinweg nicht verändert haben sollen, wie es der Autor sieht. Es ist von daher der ganz normale sarkastische Ton, den Gerhard Armanski in seinem Roman verwendet, um Sachverhalte aufzudecken. Die Beschränktheit des eigenen, kleinen Lebens wird versus der Pracht des bestehenden, großen Tempels sowie der Macht der Götter gestellt, sei es im alten oder im modernen Ägypten. „Gott ist groß“ auch im heutigen islamischen Ägypten. Der Glaube an ihn ist verbunden "im Denken des einfachen Menschen mit seiner Überlegenheit und seinen Gaben.

Horus, der die Feinde des Sonnengottes Re besiegt, nachdem Re selbst ihn mit der Sonnenscheibe zwischen seinen Flügeln verstärkt hat, wird von Re damit belohnt, dass seine Gestalt in allen Tempeln auf den Reliefs eingraviert wird. ${ }^{5}$ Er wird dadurch aligegenwärtig und darauf ist sein Diener Hor-Heb, der gleichzeitig Priester ist, stolz. Abderrachman versteht heutzutage zwar nicht mehr die Bedeutung dieser für ihn heidnischen Götter, aber er weiß, dass ihre Größe ibm nutzt und er meint zu glauben, dass Allah mit ihm ser. Er begleitet ihn bis an sein tragisches Ende, als er durch eine Kalesche am Ende des Romans tödlich verunglückt.

Der Roman leitet in seinem ersten Teil mit der zentralen Frage nach der Rolle der Götter ein, worin sich weniger eine Glaubenskrise erkennbar macht - wie wir sie heutzutage kennen -, sondern es ist eher ein Infragestellen der Vielfalt der Götter und ihrer Funktionen aus heutiger Sicht und möglicherweise auch im damaligen Bewußtsein des

\footnotetext{
${ }^{5}$ Die Information zur Legende hat hier ebenfalls dic Ägyptologin Mey Zaki Ibrahim gegeben.
} 
Priesterstandes. An dem Glauben an sich wird dabei nicht gerüttelt, da die Ägypter - anders als die Griechen - es gelernt haben, sich Gott oder den heiligen Göttern zu ergeben. Demnach stellt sich Hor-Heb die zweifelnden Fragen und beantwortet sie selbst in einer Art Selbstgespräch, ohne daß sich ein echter Zwiespalt dadurch bei ihm erweist. Er denkt:

Im Kollegium sagen sie, daß wahr nur ist, was den Gott erfreut und von ihm stammt. Aber welchen Gott? Ich gehe so of durch den inneren Tempelumgang und sehe an den Wänden, wic Horus die Feinde Res erlegt, hochaufgerichtet und siegreich, ohne daß auch nur seine Krone verrutschte. In Abydos verehren sie andere Gotter, ebenso große und strahlende, und in Alexandria wieder andere oder dieselben in anderer Bedeutung und Gesellschaft. Ich weiß, ich sollte nicht zweifeln. Vielleicht bin ich nur zu schwach, ich, ein Priester des Horus von Edfu dritten Grades. Wie komme ich dazu, auch nur eine Frage zu stellen? Hat es doch immer genigt. dem Gott zu dienen wie Staub der Erde. Die Wahrheit als eine, wie die Griechen denken, gäbe es gar nicht, sagen die Väter, sie habe viele Gesichter, wie ein Gesicht Falten werfe. (Edfu 13)

Dieser grundsätzliche Unterschied zwischen dem widerspruchslosen, gehorsamen Dienen der Ägypter und dem fragenden, zweckmäßigen Dienen der Griechen unterstreicht die Differenz, die sich offensichtlich in der ägyptisch-orientalischen Mentalität und der griechisch-europäischen Denkweise bis heute zeigt - olne diese bewerten zu wollen. Die Griechen suchen die Wahrheit an und für sich und für die Ägypter ist die Wahrheit von Gott gegeben. Sie braucht nicht gesucht zu werden, denn ihre Grundsätze sind klar. Der Glaube und die Heiligkeit prägen das Leben, die Mentalität und die Verhaltensweise der Ägypter. Glaube ist nicht durch logisches Denken ersetzbar, höchstens durch ibn zu ergänzen. Aber auch dessen ist der Glaube nicht bedürftig.

Eine grundlegende Perspektive des Erzählens, auch wemn sie nicht durch den Er-Erzähler in den Vordergrund rückt, ist demnach der Blick der Ägypter auf die Griechen und die Sichtweise der Griechen auf die Ägypter in bezug auf die unterschiedliche Denkweise. In dieser wechselhaften, variierenden Perspektive erfährt primär die ägyptische 
Mentalität und der altägyptische Glaube seine Entdeckung und Entlarvung, denn um ihn geht es in diesem Roman, nicht um den europäischen, der auf den griechisch-antiken Kult basiert. Die Gegenüberstellung von ägyptisch and griechisch dient der Enthüllung ägyptischer Denk- und Gefühlswelten im Alten Ägypten. Zı dieser Zeit, der ptolemäischen, einer ganz besonderen Zeit des pharaonischen Reichs, treffen zwei Kulturen aufeinander. Die Griechen sind die Herren im Lande, auch wenn sie sich selbst zu Pharaonen ernennen müssen, um Glaubwürdigkeit zu erhalten.

Es sind diese Griechen, denen jetzt das Land gehört, aber das ändert ja nichts am Lauf der Dinge, [...]. Es sind die Griechen, ihre grübelnden Männer im fernen Alexandria, die Philosophen, die es wieder von anderen gehört haben und behaupten, die Gotter wohnten im Geist und im Stein nur ihr Abbild. Aber ist der Geist denn etwas anderes als die Summe des Gewesenen, viel-anschaulich und faßbar, wenn auch nicht für jedermann? (Edfu 22)

Für die Griechen scheint es keinen Widerspruch zwischen Geist und Materie zu geben, im Gegenteil, sie ergänzen sicl sogar oder müßten es zumindest. Die Ägypter der damaligen Zeit sehen das etwas anders: sie brawchen etwas Faßbares bzw. Konkretes als Beweis des Göttlichen. Durch den griechischen Einfluß wird dies aber revidiert, wenigstens beim Protaganisten Hor-Heb. Er ist in der Lage zu abstrahieren und eine plausible Geschichte zu erzählen, als er den Status seines Gottes, Horus von Edfu, in der Priesterversammlung festigen und erhöhen muß. Die ,Realität aus Stein’ gewinnt erst dann an Bedeutang, wenn sie mit einer Legende von Größe und Pracht verbunden ist bzw. wird.

"Was soll ich euch von der weltumfassenden Herrin Hathor erzählen?“ [...]
"Mutter der Menschen und Inbild aller Frauen. Tochterfreude und würdige
Gemahlin des Re, verfügend über alle weiblichen Reize." [...] „Vor allem aber",
[...], „ist sie die Gemahlin des hochherzigen und mutigen Horus von Edfu, der
aus Behedet im Delta stammt, den sie alljährlich von Dendera aus zur Hochzeit
besucht. Beide duften sie vor Liebe in ihrem Gemach auf dem höchsten Punkt des
,Hauses des Horus', welchen Namen sie alsdann trägt. [...] Hor-Heb ist
erleichtert, hat er doch gefürchtet, mit seiner griechelnden Logik, ohne
weitschweifige mythische Ausschmückung, und noch immer wirr genug, die 
Heiligen Väter, die gewohnt sind, wie Spinnennetze und nicht wie Sonnenstrahlen zu denken, vor den Kopf zu stoßen. (Edfu 62)

In dieser Situation gelingt es Hor-Heb, trotz der gemischten Verhältnisse, und seinem schon von den Griechen durchdrungenes Denken, seine ägyptischen Wurzeln zu behalten. Beachtenswert ist hier die Schilderung der Denkweise der höheren Priester, die sich - wie hier beschrieben nicht gerade durch Geradlinigkeit und Klarheit auszeichnet, kein fremdes Merkmal für die ägyptische Gesellschaft. Dieser Zug wird nicht gerade als positiv dargestellt, denn der Unterschied ist groß zwischen einem Denken, das wie „Spinnennetze“ (pharaonisch-orientalisch) oder wie "Sonnenstrahlen“ (antik-okzidentalisch) empfunden wird. Dieser Kritik Arınanskis geht - ausgewogen wie er beide Kulturen gegenüberstellt ein Lob voraus und zwar in bezug auf die Rolle der Frau. In der Beschreibung der'Hathor von Dendera als Frau des Horus von Edfu, aber auch als „Mutter der Menschen“ kommt er der Vorstellung der Frau im Islam sehr nahe. Auch die Frau als Tochter, Sclhwester, Geliebte und Ehefrau ist ein im modernen Ägypten wiederkehrendes Bild. Denn die Frau wird als Inbegriff aller Mütterlichkeit und Weiblichkeit betrachtet. Sie besitzt die dazughörigen Eigenschaften und erfüllt ihre Rollen unverzüglich. Daraus resultiert ihr hoher Stellenwert bis heute. Daß „Hathor" stellvertretend für alle Frauen diese Stellung verkörpern könnte, wird hier deutlich. Sogar Hathor ist nicht nur eine Göttin, sondern eine Frau und - wie ihr Name bedeutet - ist sie das „Haus des Horus“. Sie ist eine Göttin, die ihrem Mann ein zu Hause ist.

Zur weiteren Abhebung beider Denkarten voneinander, der ägyptischen von der griechischen, ist die rächste Station des Autors ein Vergleich zwischen Theben und Alexandria. Im Text wird der Kontrast zwischen Theben ais Zentrum des altägyptischen Götterkults und Alexandria als Regierangssitz der Griechen und neues Kultzentrum 
besonders hervorgehoben. Fasziniert von Alexandria berichtet der Protagonist, auch wenn er letztendlich die Stadt für schön, aber nicht für heilig hält:

Was für eine Stadt. [...]
Der geniale Blick des großen Alexander hatte erkannt, daß aus dem Fischerdorf
[...] infolge seiner einmaligen Lage ein guter Platz für eine geräumige Hafenstadt
mit geschützter Bucht werden konnte. Seine Nachfolger, die ersten Ptolemäer,
hatten das aufs prunkvollste umgesetzt. Nun ist die Stadt mit mehreren Häfen
gesehen, deren Glanzstück der Leuchtturm auf der Insel Pharos bildet, und durch
den Nilkanal mit dem Mareotis-See verbunden. Sie birgt eine Million Einwohner
- Ägypter, Griechen, Juden, Levantiner und viele andere, die sich mischent.
Doppelnamen kommen häufig vor. Die Vielfalt macht das Zusammenleben aber
auch konflikthaft und leicht erregbar. (Edfu 72) Aber Alexandria wäre keine heilige Stadt. (Edfu 73)

Die Vielfältigkeit der Rassen macht Alexandria zur Weltstadt, weltoffen und empfänglich für neues, sich wandelndes Denken. Ob dieses Denken von Nutzen oder eher schädlich für die ursprünglichen Einwohner des Landes ist, sei dahingestellt. Die Anwesenheit von Fremden, jedoch nicht von Fremdherrschern, wurde dennoch mit Toleranz und Akzeptanz in Alexandria empfangen, was Alexandria bis heute als Stadt und Bewohner moch ist. Daran versucht der Verfasser zu erinnern. Seine Hauptfigur, Hor-Heb, kann sich damit weniger anfreunden. Heilig ist die Stadt für ihn auf keinen Fall, ein Zeichen dafür, daß im alten sowie im modernen Ägypten die Beṿölkerung des Landes, allen voran Intellektuelle und Geistliche, keine Fremdherrschaft dulden, geschweige denn respektieren. Nur als Gäste sind Fremde willkommen. Die Durchdringung ägyptischer Lebens-und Denkweisen durch Fremde ist schon immer gescheitert, Demnach stellt Alexandria für Hor-Heb das Chaos, das Fremde dar während Edfu das Geordnete, Vertraute ist.

Was Hor-Heb die Orientierung erschwert, ist das Wirrwarr der Seitengäßchen, [...]. Farben und Gerüche, das Geschrei der Ausrufer und die Fetzen vielerlei Sprachen benehmen ihm schier den Sinn. Mit gesenktem Kopf nimmt er gerade noch die Schemen von Frisuren und Gesichtern wahr, griechische Locken über weißer Haut oder ägyptische Pagenköpfe mit olivfarbenem Grund. Die Säume der 
Frauenkleider kann er sehen, die um die Hüfte [...] gerafft sind. Die Schönheit der Alexandrinerinnen, und zwar jeder Herkunft, ist berühmt, und auch von ihrer Zugänglichkeit erzählen sich die Männer Frohes. Obwohl ihnen das griechische Familienrecht weitaus weniger Rechte zuräumt als den Ägypterinnen, zeigen sie sich selbstbewußt, ja herausfordernd. Vielleicht nur hier auf der Straße, denkt Hor-Heb. Die Ägypterin bewahrt das Haus. Das Leben tritt aus einem Gefäß hinein und ins Grab aus. Bedeutet der Name Nephtys nicht „Herrin des Hauses“, und ist nicht Hathor das „Haus des Horus"? Auch Isis ruft ihren toten Gemahl in Haus und Schoß zurück. (Edfu 74)

Die Bewahrung der Tradition ist ein Gedanke, den die Hauptfigur hier weiterführt. Hor-Heb fühlt sich verwirrt in der neuen Stadt. Ihn wundern die vielen Gesichter, die er auf den Straßen Alexandrias sieht. Diese Vielfalt ist er in Theben nicht gewohnt. Die Straßen der neuen Hauptstadt Ägyptens, die die Griechen bauten, sind laut und bunt. Menschen verschiedener Herkunft streiten und vertragen sich, aber leben letztendlich zusammen. Für ihn ist es eine Welt, die ihn überrascht, Neues bietet, aber nicht seine Welt ist. Dies wird noch klarer in seinem Gedankengang über die alexandrinischen Frauen. Er preist die Geborgenheit und die Häuslichkeit, die die ägyptische Frau auszeichnen. Im Vergleich zur alexandrinischen oder griechischen beweist sie damit ihre Traditionalität. Trotz der Fortschrittlichkeit ägyptischen Familienrechts, sçbon zur damaligen Zeit, hat dies nicht zur Folge, dass ägyptische Frauen weniger sittlich sind, im Gegenteil, sie wissen damit umzugehen. Hier macht sich eine Kritik nicht nur am damaligen griechischen Recht bemerkbar, sondern auch am beutigen ägyptischen Recht. Die Aussage ist, daß mehr Rechte für die Frauen nicht gleich ihre Freizügigkeit bedeuten, sondern $\mathrm{da} \beta$ Frauen, in diesem Falle die Ägypterinnen, ihre zugestandene Freiheit rechtmäßig und verantwortungsvoll handhaben können. Die Wertschätzung der Mentalität ägyptischer Frauen, die ihr zu Hause vor allem vorziehen, findet hier ihren Ausdruck nebst der Erteilung ihrer Rechte. 
Gedanklich zurück in seiner oberägyptischen Welt, sucht Hor-Heb nach dem Ursprung der Menschheit und des Glaubens, und wirft seine alexandrinischen Zweifel ab. Ausschlaggebend für ihn - wie für jeden Ägypter seit eh und je - ist der Bezug zum Nil und die Verbundenheit mit dem Nîltal. Er macht sich folgende Gedanken darüber: „Hängt nicht jeder Tempel über den Heiligen See an der Nabelschnur des Nils?" (Edfu 118). Keineswegs bildet jeder Tempel eine in sich geschlossene Einheit, sondern sie sind alle mit dem Ursprung der Welt, wie er von den Vorvätern und Urgroßvätern überliefert wurde, verbunden. So wie der Historiker Herodot später feststellte: Ägypten ist die Gabe des Nils! Ohne diese Gabe wäre das Land trocken und unfruchtbar. So versteht auch HorHeb die Verbindung von Tempel, Nil und Land als eine Einheit. Daran können die Griechen mit ihrer Logik und ihren Theorien nichts ändern. Die Philosophie kann nicht anstelle des Glaubens treten.

Die Griechen stellen das Gesetz auf den Kopf. Nach ihnen spiegelt und regelt es, was wir wünschen. [...] Es kann nicht stimmen, was die Griechen sagen. Nichts zählt außer der ewigen Weisheit im Haus des guten Gottes. Wie sehnt er sich nach der süßen Ruhe, die sie verleiht! [...] Was vermögen dagegen die philosophischen Systeme der Griechen, die doch nur Menschenhirnen entstammen? Ihre Kraft ist die des Wissens und des Willens. (Edfu 118)

Göttliche Gebote, die das menschliche Leben ordnen, sind nicht mit menschlichen Regelungen zu ersetzen. Diese schützen nicht vor Unordnung und geben nicht die gleiche seelische Ruhe. Das Zusammenleben der Menschen in einer Gesellschaft kann nach altägyptischem Glauben nur durch heilige Weisheiten geregelt werden und verleiht damit dem Menschen Ruhe und Seeligkeit. Dementgegen stehen die vom Menschen entworfenen Gesetze, die irre führen können und vergänglich sind. Hier wird wieder die Beständigkeit im ägyptischen Glauben versus der sich wandelnden griechischen Mentalität dargestellt. Es wird darauf hingedeutet, daß durch die menschliche Gesetzgebung und die Anfechtung, des göttlichen Gesetzes im engeren Sinne 
Ungerechtigkeit und Armut initiiert, im weiteren Sinne Clıaos und Anarchic. In ihnen kann eine Parallele zur lieutigen Globalisierungstendenz gesehen werden sowie neuen Formen des Imperialismus. Die wirtschafliche Ausbeutung ist nur eine Form davon: sie hat alte Ursprünge und kann jederzeit neu aufgelegt werden, überall auf der Welt. Das Alte Ägypten ist ein Beispiel dafür, und durch die Gegenüberstellung von ägyptischem, göttlichen Glauben und griechischer, weltlicher Logik wird dies noch klarer herauskristallisiert.

Herrschen nicht .Haß und Zwist im Reich? Seufzt der Bauer nicht unter der Last, die er zu tragen hat? Brandet nicht der Aufruhr an die Häuser der Reichen und selbst an die Tempel, hinter deren Toren sich die Güter des Gottes stapeln? Das Gebot der griechischen Politik will alles verändern und verbessern und treibt doch in den Hades. Was sie mit ihm säen, ernten sie nun im Sturm, der durch das schwarze Land zieht und es verheert. Die Tage der Könige am Meer sind gezählt. Neue Herren jenseits des Meeres greifen nach dem Ruder. Wir singen unsere Gesänge einer uralten Welt, verborgen hinter Tempelmauern, während sie sich unter dem Griff der Fremden verbiegt und unkenntlich wird. Etwas anderes können wir nicht tun, bis wir irgendwann nicht mehr begreifen, was wir singen, weil es nicht mehr das Gesetz der göttlichen Welt ist. (Edfu 118f)

Der Aufruhr wendet sich im Lande, seitdem Gottes Gesetz nicht mehr absolut herrscht, gegen die wirtschaftlichen, politischen und geistlichen Eliten im Land. Das Gesetz der Griechen hat Verbesserung versprochen, konnte dies aber nicht verwirklichen. Es ist wichtig, Altes und Tradiertes nicht abzulehnen, olne es geprüft zu haben und es besser machen zu können - so die Vorstellung Hor-Hebs. Denn auch Neues hat seine Probleme. Eigentlich scheint es dem Protagonisten schon fast so, als ob es eine heilere Welt gab, als "das Gesetz der göttlichen Welt“ noch befolgt wurde. Er befürchtet, daß er irgendwann seinen göttlichen, heiligen Gesang nicht mehr verstehen wird, da seine Umwelt sich für ihn bis zur Unkenntlichkeit verändert hat. Seine Gefühle sind durcheinander. Was in den Innenwänden des Tempels vor sich geht, hat sich von seiner Außenwelt abgehoben. Gottes Gesetz muß nicht erklärbar sein und man muß es eigentlich nicht begreifen, sondern nur die Ordnung bewahren 
wie man sie seit jeher kemnl. Denn egal worauf man sich bei der göttlichen Ordnung beruft, sei es Heiligkeit oder Sinn, sie sorgt für den Zusammenhalt der Menschen und für das Aufrechterhalten gesellschaftlicher Strukturen, die sowohl Ethik als auch Moral beschützen, auch wemn es eine ritualisierte Ethik und Moral ist. Denn letztendlich geht es nicht darum, welchen Gott man anbetet, da es im Endeffekt nur einen Gott gibt, und der Glaube an ihn dazu dienen soll, das Leben des Menschen zu verbessern, nicht umgekehrt. Die Idee des Monotheismus steht in diesem Zusammenhang und sie gehit dabei mit der Suche nach der Uridee des Göttlichen einher:

„Dein Gott u'nd mein Gott sind groß und andere auch, wir wissen es. Aber gibt es nicht einen, aus dem sie alle stammen und der sie dereinst zu bloßen Windlichtern in der Nacht des Unwissens machen wird?" Nun wird es gefährlich (aber immerhin kannten die alten Ägypter das Ketzereidelikt nicht), [...] „Verehren nicht die Hebräer einen einzigen Gott, wie ich in Alexandria erfahren habe, der keine anderen neben sich duldet? Ist es nicht die Uridee des Göttlichen, von der alles ausgeht? [...] (Edfu 132)

Da die altägyptische Kultur nicht gegenüberstellt, sondern eher verknüpft, bietet dies die Möglichkeit der Versöhnung aller Religionen gerade in Ägypten. Dies kann vom Verfasser als Plädoyer für die Rückbesinnung auf die Urprungsidee aller Religionen betrachtet werden. Es ist ein Aufruf zu mehr Toleranz, der gerade aus dem Land der ersten Zivilisation ausgehen kann. Es ist eine Aufforderung an die Menschlheit in dem Land, in dem diese Vielfalt an Göttern im Altertum angebetet wurde (und das allen Himmelsreligionen heilig war und ist), ein Exempel zu sehen. Denn es war schon immer ein Treff- und Versöhnungspunkt. Auch ist der erste monotheistische Gedanke im pharaonischen Ägypten mit Ekhnaton entstanden. Die Aussage des Verfassers ist, daß es letztendlich einen einzigen Gott gibt oder geben muß, auf den jeglicher Glaube zurückzuführen ist. Die Botschaft der Toleranz, des Friedens und der Seeligkeit wird hier deutlich ausgedrückt. 
Im modernen Ägypten stellt sich der Tempelwächter Abderrachınan ähnliche Fragen wie Hor-Heb, die der Religionsgelehrte des Dorfes bzw. der „Imam der Moschee“ nicht begreifen kann. Er wundert sich über die Zweifel des Wächters Abderrachman und kann sie nicht fassen. Er führt sie auf seinen häufigen Umgang mit den ausländischen Touristen zurück. Abderrachman wiederum stellt die Besuche der Touristen in Frage! Er versteht nicht, weshalb die alten Tempel und die „Götter aus Stein“ solch einen Reiz für sie haben.

Was sie im Tempel zu suchen haben und vielleicht finden, weiß er nicht, und es berührt ihn nicht. Der Ortsgeistliche hebt auch nur die Schultern, wenn man ihn fragt. Starre Figuren in Stein, die unverständlich aussschauen und herumstehen. [...] Im Schulbuch seines Sohnes hat er einmal das Bild unter der Unterschrift "Leben der Vorväter" betrachtet. Priester hat man darauf gesehen, kahlgeschoren, weißgekleidet und beleibt, wohlbefunden also. Das hat Abderrachman verstanden. So soll es sein. Aber gab es denn damals nur Priester, vielleicht auch Könige? Von den einfachen Leute, so wie er, ist in dem Buch nicht die Rede gewesen. Er kann nicht glauben, daß die Priester auch das Lande bebauten und einem Handwerk nachgingen. Der Imam in der Moschee hatte lange nachgedacht. "Es wird damals nicht anders gewesen sein als heute. Die einen ernähren und die anderen werden ernährt." Er hatte Abderrachman stirnrunzelnd angesehen. Was der für Fragen stellte! Das muß vom Umgang mit diesen Ungläubigen kommen. (Edfu 45)

Das „Leben der Vorväter" leuchtet ihm nicht ganz ein. Es kann nicht sein, daß die Vorväter nur Könige und wohlhabende Priester waren. , Wo bleibt da das Volk?' ist die Frage, die sich Abderrachman stellt. Ähnlich wie Hor-Heb zweifelt er, aber auf seine Art. Beide kritisieren soziale Ungerechtigkeit. Denn die „einfachen Leute“ sind es, die in der Regel das Land „ernähren“. Dies ist nicht Gott gewollt, und schon gar nicht ein Gebot Gottes, sondern es ist eine geschichtliche Wirklichkeit, die wohl zugleich universell ist. Der Umgang mit den „Ungläubigen“ hat ihn einerseits zweifeln lassen, andererseits hat er ihn kritischer gemacht, je nachdem wie man das sieht. Abderrachmans Gedanken unterstützen nur die Nebenhandlung im Roman und lassen uns eine Parallele zum modernen Ägypten ziehen. Die Hauptfigur bleibt aber Hor-Heb, ein 
Priester dritten Grades des Horus von Edfu. Die ganze Handlung des Romans dreht sich primär in Zeit und Raum des Alten Ägyptens.

Der Roman weist auf die Ursprünge zweier Kulturen hin, versucht diese zu analyșieren und schließt eine Synthese zwischen zwei grundsätzlich unterschiedlichen Kulturen, auch in ihrer modernen Entwicklung, nicht aus. In der Erzählung steht dabei nicht die altgriechische Kultur im Mittelpunkt, sondern die altägyptische.

Wollte man zusammenfassen, könnte man sagen, daß voin einer tatsächlich statischen Mentalität der Ägypter nicht die Rede sein kann. Auch wenn der Autor das manchmal so darstellt, dennoch zeigen sich ebenfalls die Vorteile dieser unveränderten Mentalität, nämlich Ordnung and Beständigkeit. Anarchie ist von den Ägyptern, damals wie heute, nicht gewollt. Ein Zweifeln und Infragestellen aller ,Konstanten' ist keine ägyptische Eigenschaft, eher eine europäische; um es mit der Sprache Armanskis zu sagen: eine pharaonische und keine griechische. Der Glaube ist heilig und kann nicht angefochten werden.

Die alten Kulturen stehen repräsentativ für das unterschiedliche Denken in Ost und West; keine der beiden Kulturen wird angeprangert. Der Autor stellt dar und fragt mit, aber er urteilt nicht. Ägypten, als Standort der ersten großen Kultur der Welt, wird in detaillierten Besclureibungen der Tempel- und Götterwelt erschlossen. Dennoch bleibt es geheimnisvoll. Es behält seine Magie. Der Zauber resultiert aus der Vielfalt, aus den vielen möglichen Facetten der Götterwelt im Alten Ägypten, die trotz aller Ausführung in den Einzelheiten der Erzählung nicht ausschöpfbar sind und werden.

Gerhard Armanski gelingt es, nicht nur die altägyptische Kultur zu ergründen, sondern auch einen für Ägypter und Europäer interessanten historischen Roman zu schreiben. 


\author{
Nagla S.El-Dinndouslı \\ Dozcntin an/kommissarisclic Lcilcrin \\ der Abtcilung fïr Gcrmanistik \\ Philosophisclic Fakultıı \\ Helwan Universitt! \\ Erstc Intcrnationalc Konfcrenz. \\ der Al-Alsun Fakultalı. Universilal Minia \\ "Das Ägypıcnbild in der Weltlitcralur" \\ 14. - 15. April 20()4
}

\title{
Zusammenfassung
}

\section{Zum Glauben und zur Mentalität der Alten Ägypter in Gerhard Armanskis Roman Die geflïgelte Sonne in Edfu. Roman aus dem prolemä̈ischen ïgypten.}

\begin{abstract}
Dieser im Jahre 2000 erschienenc Roman steht zwar in der Tradition des historischen Ägypten - Romans, doch erzählt er die Geschichte der Menschen am Nil im Ralımen der altägyptischen Götterwelt aus einer subjektiven, fiktiven Perspektive. Episoden aus dem modernen Ägypten werden in ihn eingeblendet und bilden eine Parallcle zu der angeblich unveränderten, statischen Mentalität der Ägypter. Aufrallig ist auch, dass der Roman niche nur von altägyptischen Glaubensweisen und Denkmustern handelt, sondern sich auch zu antiken griechischen Vorstellungen äusser:, dic im ptolemäischen Ägypten in seiner kosmopolitischen Hauptstadt Alexandrien Verbreitung fanden. Pharaonische und hellenistische Kultur repräsenticren hier östliches und westliches Denken. Aber der Autor stellt es nur dar, ohne es zu bewerten und er schließt darüber hinaus eine Synthese zwischen den beiden unterschicdlichen Kulturen nicht aus.

Da das Buch noch relativ neu ist, und keine Forschungsliteratur darüber existicrt, wird in dem vorliegenden Beitrag cine durchgängig werkimmanente Textanalyse vorgenommen.

Im Mittelpunkt des Romans stelt Hor-Heb, ein Priester des Dritten Grades in HorusTempel von Edfu. Parallel dazu erscheint der moderne Ägypter Abderrachman, cin Wäcliter des alten Tempels, der die ausländischen Touristen aus sciner Perspektive betrachtet. Die Beschränktheit ihres cigenen, kleinen Lebens steht im Gegensatz, zu Macht und Pracht der Religion. Sowohl ollor-Heb als auch Abderrachman kritisieren soziale Ungerechtigkeit und müssen sich mit Glaubenszweifeln auscinander setzen.

Sic finden jedoch den Hauptsinn in der göttlichen Ordnung, weil sic durch Ethik und Moral die gesellschaftlichen Strukturen aufrecht erhält.Göttliche Gebote, dic das Leben auf Erden ordnen, sind nicht durch menschliche Regeln zu ersetzen.
\end{abstract}




$$
\begin{aligned}
& \text { المزتَمر الدولي الأول لكلية الألسن }
\end{aligned}
$$

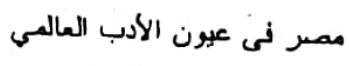

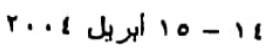

\section{ملخص}

\section{عقيدة و اساليب تفكير القدماء المصريين فى رواية جرهارد ارلسكى وعنوانها " الشمس ذات الأجنحة في ادفو " \\ رواية تتناول مصر البطالمة}

تقع هذه الرواية التي تم نشرها فى عام . . . فى نطاق الرواية المصرية التاريخية وتتتــاول

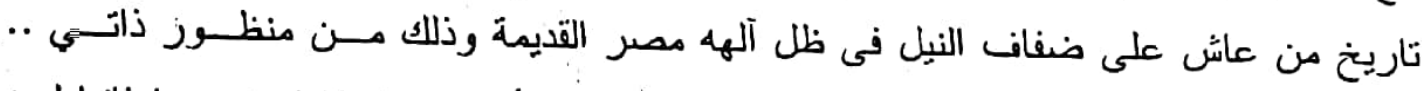

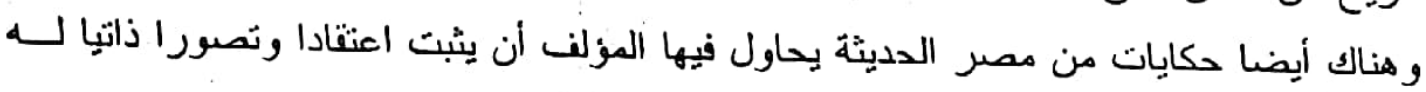
يرى بمقتضاه العقلية المصرية عقلية غير متطورة لم تثغير على مر العصور . ومن الجدير بالذكر أن هذه الرواية لا تتتاول فقط معثتدات المصريين القدماء وطرز تفكيــرهم ولكنها نتتاول أفكار إغريقية قديمة انتشرث فى مصر البطالمة فى عاصمتها آنذاك الإسكندرية ذات الطابع العالمي ..

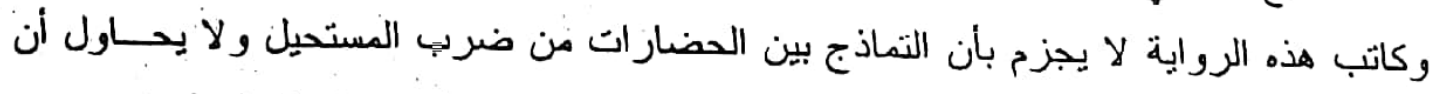

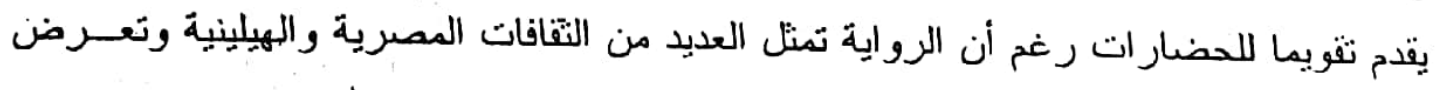

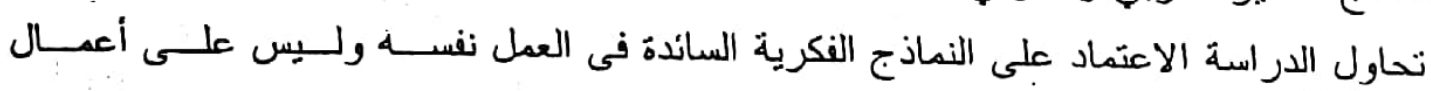
نماذج التفكير الغربي و الشرقي ..

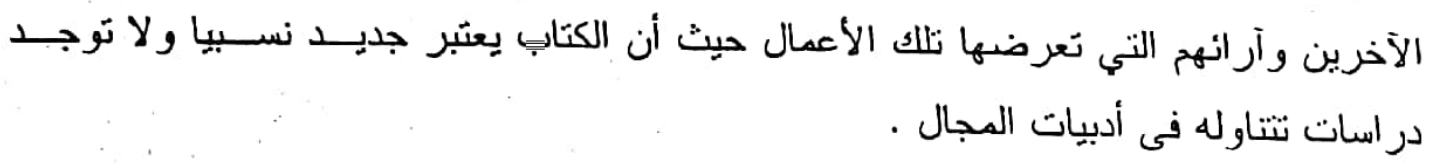
تعتبر النخصية الرئيسية فى هذا العمل هى شخصية هور هب وهو قسبِ ليس هن الدرجات

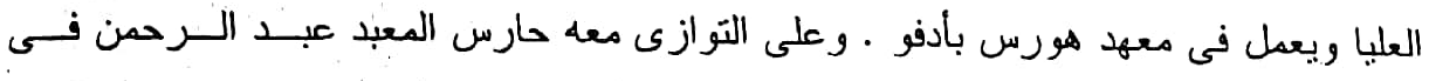

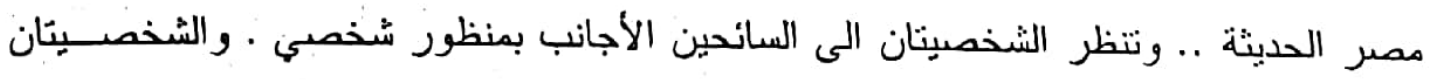

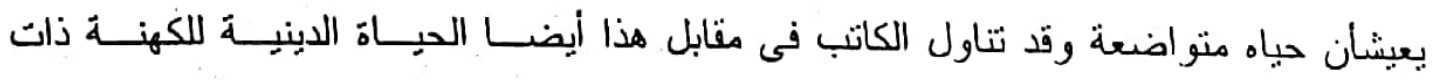

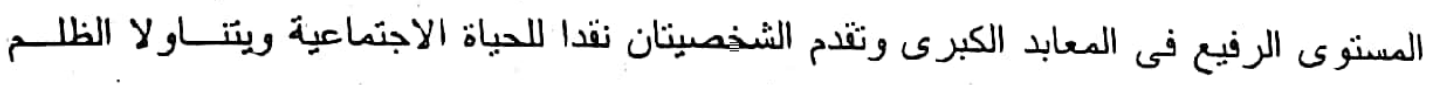




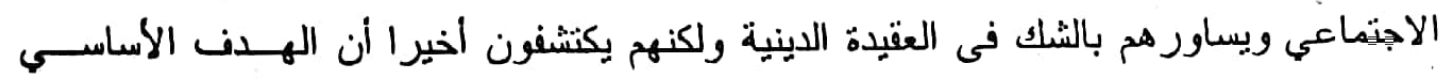

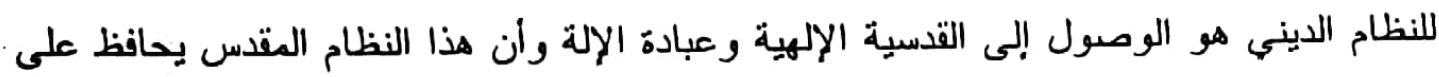

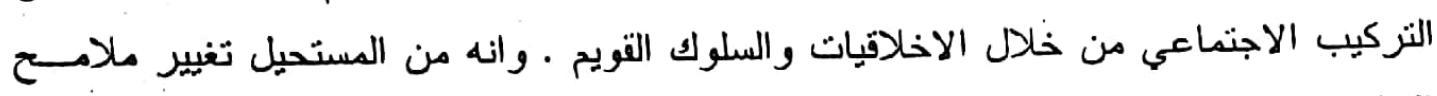

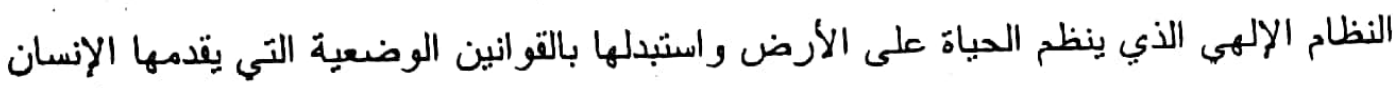

